\title{
Endogenous heparin levels in the controlled asthmatic patient
}

\author{
Hajierah Davids, Ayesha Ahmed, Ari Oberholster, Christo van der Westhuizen, Mervyn Mer, Ivan Havlik
}

Background. Since heparin possesses anti-inflammatory properties, it is hypothesised that asthmatic patients have decreased levels of circulating heparin compared with healthy individuals.

Design. We compared endogenous heparin levels in controlled asthmatic patients (53 adults) from the Asthma Clinic at Johannesburg General Hospital with those of healthy controls (26 adults) from the general population. Heparin levels in the blood samples were tested using the Chromogenix Coatest Heparin kit.

Result. The blood of the patients contained significantly lower levels of endogenous heparin compared with that of the healthy individuals, indicating that the anti-inflammatory properties afforded by heparin are absent in these patients.

S Afr Med J 2010; 100: 307-308.
Levels of circulating endogenous heparin in patients with diseases associated with inflammatory processes, such as asthma, are still unknown. Since heparin displays antiinflammatory properties, it is expected that the inhibitory effects on inflammatory mediators are reduced at low levels of endogenous heparin. ${ }^{1}$ We present evidence supporting the hypothesis that asthmatic patients have lower levels of circulating endogenous heparin than healthy individuals have.

Since 1955, endogenous plasma heparin has been demonstrated to be a normal constituent of blood, with concentrations ranging from 153 to $177 \mathrm{mIU} / \mathrm{ml}$, and its levels were found to be independent of gender or age. ${ }^{2}$ Discrepancies in the concentrations of endogenous heparin reported by various research groups are thought to result from the different quantitative methods or isolation procedures used.

Inhaled heparin has shown potential as an anti-asthmatic agent. In the airways, arginine availability determines the production of the bronchodilator, nitric oxide. Changes in arginine homoeostasis may therefore cause airway hyperresponsiveness and remodelling of the airway wall. Inhaled heparin was found to decrease airway hyper-responsiveness in early asthmatic reactions and peroxynitrite-mediated hyperresponsiveness in late asthmatic reactions induced by a variety of stimuli in guinea pig and rat models of allergic asthma, as well as in asthmatic patients. ${ }^{3}$

The anti-allergic activity of inhaled heparin was found to be independent of its anticoagulant properties, where an inverse relationship between the molecular weight of heparin and anti-

Department of Pharmacy and Pharmacology, University of the Witwatersrand, Johannesburg

Hajierah Davids, BSc, BSc (Hons), MSc, PhD

Ayesha Ahmed, BSc, BSc (Hons)

Ivan Havlik, MSc, PhD

Department of Pulmonology and Critical Care, University of the Witwatersrand Ari Oberholster, Nat Dip Pulmonol

Christo van der Westhuizen, Nat Dip Pulmonol

Mervyn Mer, MB BCh, Dip PEC (SA), FCP (SA), MMed (Int Med), FCCP, FRCP (Lond) allergic activity exists. ${ }^{4}$ In patients with a history of exerciseinduced asthma, inhaled heparin did not affect respiratory parameters, partial thromboplastin time or heart rate, and prevented post-exercise bronchoconstriction. In addition, no changes to bronchoconstrictor responses to histamine were noted. These findings suggested that heparin activity was associated with altering mediator release and not as a direct effect on smooth muscle. ${ }^{5}$

Our study was aimed at determining the levels of circulating plasma heparin in asthmatic patients compared with healthy individuals, based on the proposed role of heparin as an antiasthmatic agent.

\section{Methods}

Approval was obtained from the Human Ethics Committee of the University of the Witwatersrand for this study, which was conducted in collaboration with the Asthma Clinic at Johannesburg General Hospital.

A total of 53 controlled asthmatic patients from the Asthma Clinic were enrolled, and 26 healthy individuals from the general public agreed to participate.

Individual diagnoses of asthma were established according to spirometry testing: forced vital capacity, forced expiratory volume in one second and forced expiratory flow readings. To avoid interference of too many confounding variables, patients with a history of multiple illnesses were not approached to participate. Confirmed asthmatic patients with associated diseases such as allergic rhinitis and gastro-oesophageal reflux disease (GORD) were not excluded from the study.

Exclusion criteria for healthy individuals included past and present smokers, significant abusers of alcohol, hospitalisation in the previous year, taking medication (anticoagulants), and having suffered from the common cold in the preceding 3 months.

Blood was sampled from the volunteers after they had given signed, informed consent. Heparin levels in the plasma were determined using the Chromogenix Coatest Heparin kit as per the manufacturer's specifications. This photometric kit uses the chromogenic substance S-222 (Bz-Ile-Glu-(g-OR)-Gly-Arg- $p$ NA. $\mathrm{HCl}$ ) as substrate. 


\section{Original Articles}

\section{Data analysis}

Heparin levels are reported as mean $\pm \mathrm{SD}$ of three experiments. The $t$-test was used to compare data, using GraphPad Prism version 3.0 at a $95 \%$ confidence limit.

\section{Results and discussion}

A total of 53 asthmatic patients were enrolled ( 9 males and 44 females) and classified as suffering from mild persistent, moderate persistent or severe persistent asthma, based on their spirometry readings. Very low plasma heparin concentrations were determined for all asthmatic patients $(2.231 \pm 0.001 \mathrm{mIU} /$ $\mathrm{ml}$ ), these levels being on the threshold of the sensitivity of the assay kit. No significant differences in endogenous heparin levels were noted among the age groups $(p=0.480)$ or the genders $(p=0.786)$.

Of the 53 patients, $2(3.8 \%)$ were current smokers and 9 $(16.9 \%)$ were past smokers, 30 (56.6\%) were non-smokers, and the remaining $22.6 \%$ did not respond to this question.

A total of 15 (28.3\%) patients either did not present with other illnesses or did not respond to this question, while 15 (28.3\%) suffered from allergic rhinitis, and $11(20.8 \%)$ suffered from GORD, of whom 3 also suffered from allergic rhinitis. Of the 15 patients suffering from hypertension, 1 suffered from allergic rhinitis, while 5 also suffered from GORD.

Medications prescribed included Budeflam (budesonide), Asthavent (salbutamol) and Beconase (beclometasone), among others.

A total of 26 healthy volunteers were recruited, of whom $10(38.5 \%)$ were male. Our results showed that endogenous heparin levels did not differ significantly between the genders ( $p=0.859)$ or among the age groups $(p=0.546)$, supporting the findings by Engelberg. ${ }^{1}$ Based on these findings, we did not attempt to gender- and age-match the healthy volunteers to the asthmatic patients.

Average plasma heparin levels in these volunteers $(451.0 \pm 0.0248 \mathrm{mIU} / \mathrm{ml})$ were significantly higher $(p<0.001)$ than those of the asthmatic patients.

Significantly higher levels of endogenous 'heparin-like' substances were found in the plasma of atopic asthmatic patients, as opposed to non-allergic, non-asthmatic controls. It has been suggested that heparin was released to decrease eosinophil recruitment into allergic inflammatory sites and to minimise the degree of tissue damage resultant of cationic proteins derived from the leucocyte. ${ }^{4}$ These effects may be the underlying mechanism in the reduction of exercise-, histamineand allergen-induced bronchoconstriction. ${ }^{6}$

These preliminary results indicate significantly decreased endogenous heparin levels in the asthmatic patient, thereby potentially decreasing anti-inflammatory effects. As it is not clear whether the decreased levels of heparin are a result of the medication (in particular $\beta_{2}$-agonists) being administered, or a manifestation of the disease itself, our research is an ongoing endeavour to clarify this aspect.

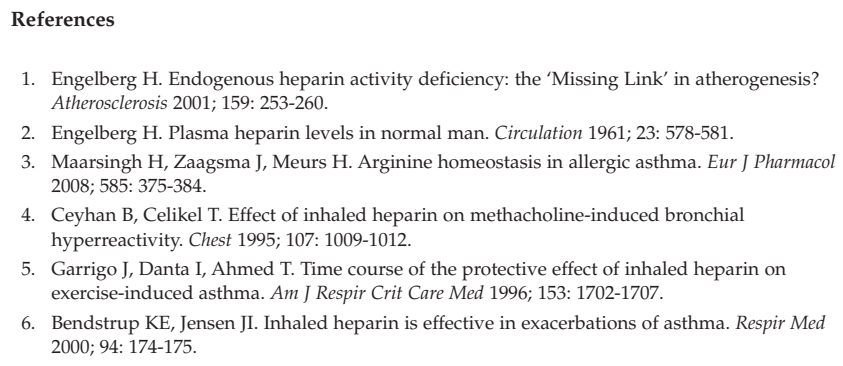

Accepted 31 August 2009 